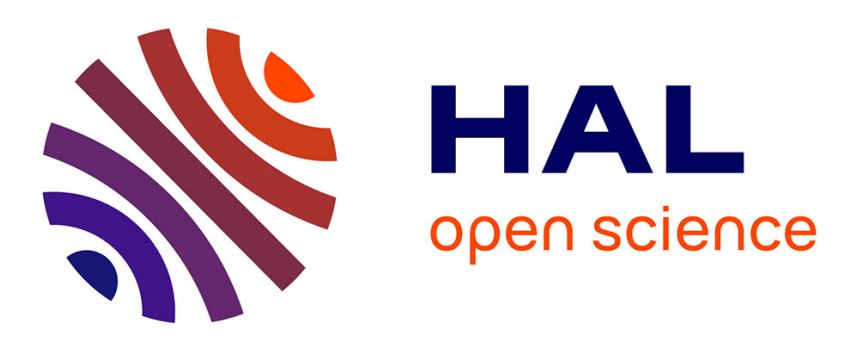

\title{
Interprétation physique des ondes de Floquet se propageant dans un milieu multicouche périodique anisotrope
}

Catherine Potel, J.-F. de Belleval

\section{- To cite this version:}

Catherine Potel, J.-F. de Belleval. Interprétation physique des ondes de Floquet se propageant dans un milieu multicouche périodique anisotrope. Journal de Physique IV Proceedings, 1994, 04 (C5), pp.C5-685-C5-688. 10.1051/jp4:19945146 . jpa-00252824

HAL Id: jpa-00252824

https://hal.science/jpa-00252824

Submitted on 1 Jan 1994

HAL is a multi-disciplinary open access archive for the deposit and dissemination of scientific research documents, whether they are published or not. The documents may come from teaching and research institutions in France or abroad, or from public or private research centers.
L'archive ouverte pluridisciplinaire HAL, est destinée au dépôt et à la diffusion de documents scientifiques de niveau recherche, publiés ou non, émanant des établissements d'enseignement et de recherche français ou étrangers, des laboratoires publics ou privés. 


\title{
Interprétation physique des ondes de Floquet se propageant dans un milieu multicouche périodique anisotrope
}

\author{
C. POTEL et J.-F. DE BELLEVAL
}

Université de Technologie de Compiègne, Centre de Recherches de Royallieu, LG2mS, URA 1505 du CNRS, BP. 649, 60206 Compiègne cedex, France

\begin{abstract}
The aim of this paper is to better understand the correspondence between the classical plane waves propagating in each layer of an anisotropic periodically multilayered medium and the Floquet waves. The last are linear combinations of the classical plane waves. Their wave number is obtained from the eigenvalues of the transfer matrix of one cell of the medium. We have defined a Floquet polarisation which varies with its position in the periodically multilayered medium. This allows us to define a Floquet waves displacement by analogy with the one of the classical plan waves, and to verify the equality of the two displacements at any interface separating two layers. The periodically multilayered medium is then equivalent to a dummy material, and one can draw dispersion curves. In low frequencies range, when the relation between the Floquet wave numbers and the frequency is linear, the multilayered medium can be homogenised; the Floquet polarisation at different interfaces tends to a limit which is the polarisation of the classical plane wave in the homogenised medium.
\end{abstract}

\section{1 - INTRODUCTION}

L'étude de la propagation dans un multicouche anisotrope périodique se fait maintenant classiquement par la méthode des matrices de transfert $[1,2,3]$. Cette méthode permet d'exprimer les amplitudes de déplacement des ondes dans une couche du multicouche en fonction de celles dans la précédente. Si l'on appelle supercouche la cellule de base d'un multicouche anisotrope périodique, c'est-à-dire l'assemblage de $Q$ couches anisotropes distinctes, on peut alors calculer la matrice de transfert d'une supercouche. Les valeurs propres de cette matrice font intervenir les lenteurs de Floquet, reliées aux nombres d'onde de Floquet $[4,5,6,7]$. Les ondes de Floquet correspondent aux modes de propagation dans un multicouche périodique infini. Ce sont des combinaisons linéaires des ondes planes classiques se propageant dans chaque couche. Nous appelons ondes planes classiques les ondes planes, modes du milieu infini de mêmes caractéristiques que la couche considérée. On peut alors considérer le multicouche périodique fini comme un milieu équivalent dans lequel se propagent les ondes de Floquet. Le passage des amplitudes de déplacement des ondes planes classiques à l'interface supérieure de la première couche d'une supercouche à celles des ondes de Floquet à la même interface se fait par l'intermédiaire de la matrice des vecteurs propres de la matrice de transfert. Le but de cet article est de mieux comprendre la correspondance entre les ondes planes classiques et les ondes de Floquet. Nous définirons d'abord les amplitudes de déplacement des ondes de Floquet à une interface séparant deux couches successives, puis un vecteur polarisation de l'onde de Floquet $(\beta)$ à cette interface. On peut alors définir le déplacement des ondes de Floquet par analogie avec celui des ondes planes classiques. La notion de milieu équivalent au milieu multicouche est donc plus forte, et on peut tracer des courbes de dispersion, un cas intéressant étant celui du comportement du multicouche dans le domaines des grandes longueurs d'onde. 


\section{2- DÉFINITION D'UN VECTEUR POLARISATION DE FLOQUET}

Le milieu est un multicouche périodique, constitué d'un empilement de $\mathrm{P}$ supercouches, chacune constituée de l'assemblage de $\mathrm{Q}$ couches anisotropes et d'épaisseurs a priori distinctes. Nous étudions la propagation acoustique des ondes générées par une onde incidente oblique monochromatique de pulsation $\omega$, se propageant dans le milieu supérieur et contenue dans un plan perpendiculaire aux interfaces.

Définissons : $\quad q$ le numéro de la couche dans une supercouche $: 1 \leq q \leq Q$

$\mathrm{p}$ le numéro d'une supercouche $: 1 \leq \mathrm{p} \leq \mathrm{P}$

$[\Phi]$ la matrice de transfert d'une supercouche

Les ondes de Floquet sont des combinaisons linéaires des ondes planes classiques se propageant dans chaque couche du multicouche. Le passage des amplitudes de déplacement des ondes planes classiques à celles des ondes de Floquet à l'interface séparant deux supercouches se fait par l'intermédiaire de la matrice des vecteurs propres de la matrice de transfert [ $\Phi]$ d'une supercouche. Or on peut aussi définir une matrice $[\Phi q]$, matrice de transfert permettant de passer des amplitudes de déplacement des ondes dans la couche $q$ d'une supercouche $\mathrm{p}$ en fonction de celles dans la couche $\mathrm{q}$ de la supercouche suivante. La matrice $[\Phi]$ définie précédemment est alors $\left[\Phi^{1}\right]$.

Toutes les matrices [ $\left.\Phi^{q}\right]$ ont mêmes valeurs propres, reliées aux nombres d'onde de Floquet par une relation exponentielle, mais des vecteurs propres différents. A chaque interface, on obtient donc les mêmes ondes de Floquet, mais qui s'expriment par une combinaison linéaire des ondes planes classiques, différente selon la couche q.

Définissons : $\quad[\Xi 9]$ la matrice des vecteurs propres de [ $\quad \Phi 9]$.

$\{F p, q\}$ le vecteur colonne $(6 \times 1)$ des amplitudes de déplacement des ondes de Floquet dans la couche $q$ de la supercouche $\mathrm{p}$.

$\left\{A^{p}, q\right\}$ le vecteur colonne (6x1) des amplitudes de déplacement des ondes planes classiques dans la couche $q$ de la supercouche $p$.

On a alors :

$$
\left\{\mathcal{A}^{\mathrm{p}, \mathrm{q}}\right\}=\left[\boldsymbol{\Xi}^{\mathrm{q}}\right]\left\{\mathrm{F}^{\mathrm{p}, \mathrm{q}}\right\}
$$

Dans toute la suite, on considérera un milieu multicouche périodique dans lequel ne se propage qu'une seule onde de Floquet repérée par l'indice $(\beta)$. Parmi les six ondes de Floquet, le vecteur $\{F \mathrm{~F}, q\}$ a donc une seule composante $(\beta)$ non nulle. En effet, en choisissant des conditions aux limites judicieuses, on peut arriver à n'avoir qu'une seule onde de Floquet dans le milieu multicouche. Le système étant linéaire, on peut retrouver la propagation réelle par superposition de tous les "états" ( $\beta$ ).

On peut alors définir le vecteur ${ }^{(\beta)} \overrightarrow{\mathrm{P}}_{\mathrm{f}}^{\mathrm{q}}$, vecteur polarisation de Floquet à l'interface séparant la couche $\mathrm{q}$ de la couche $q-1$, comme une combinaison linéaire des polarisations du matériau constituant la couche $q$ :

$$
{ }^{(\beta)} \overrightarrow{\mathrm{P}}_{\mathrm{f}}^{\mathrm{q}}=\sum_{\eta=1}^{6} \Xi_{\eta \beta}^{\mathrm{q}}{ }^{(\eta)} \overrightarrow{\mathrm{P}}^{\mathrm{q}}
$$

où

$\eta$ est l'indice repère de chacune des six ondes planes classiques dans une couche

$(\eta) \vec{p}^{q}$ est le vecteur polarisation de l'onde $(\eta)$ dans la couche $q$

Ce vecteur polarisation de Floquet, différent selon la couche et la position dans le multicouche, permet de définir le déplacement des ondes de Floquet par un formalisme tout à fait semblable à celui utilisé dans le cas des ondes planes classiques. Afin de ne pas compliquer l'écriture, le vecteur polarisation de Floquet défini par l'équation (2) n'est pas normalisé. ( $\beta$ )Fp,q est donc l'amplitude de déplacement de l'onde de Floquet $(\beta)$, rapportée au vecteur non normalisé.

L'intérêt de cette écriture est que l'on peut définir une polarisation de Floquet qui est un vecteur complexe, à chaque interface séparant deux couches. En définissant une interface virtuelle qui sépare une 
couche en deux, tout en gardant les mêmes propriétés, on peut définir un vecteur polarisation de Floquet et donc en avoir un en tout point du multicouche. Nous avons vérifié sur un exemple que ce vecteur varie selon sa position dans le multicouche. De plus ce vecteur est complexe, même pour une onde propagative. Les ondes de Floquet ont donc pour propriété d'avoir une polarisation elliptique qui varie avec la profondeur, et donc un mouvement elliptique des particules pour un mode, même si l'onde est propagative. Ceci diffère du comportement dans un matériau homogène pour lequel, si une onde est propagative alors sa polarisation est rectiligne (sauf pour un axe acoustique). La polarisation n'est elliptique que si l'onde est inhomogène.

La propagation peut donc être décrite soit par les ondes planes classiques, soit par les ondes de Floquet, combinaisons linéaires des précédentes. La définition du vecteur polarisation permet d'exprimer ces ondes de manière analogue à celle des ondes planes classiques. Physiquement, si l'on choisit des conditions aux limites à la première interface du multicouche pour n'avoir qu'une seule onde de Floquet se propageant, on retrouvera les mêmes conditions aux limites à l'interface séparant la première superconche de la suivante, puisque les ondes de Floquet sont des modes de propagation du milieu périodique infini.

\section{3 - COURBES DE DISPERSION ET HOMOGÉNÉISATION}

On peut donc étendre la notion de milieu équivalent au milieu multicouche et donc tracer, comme pour tout milieu homogène, des courbes de dispersion et des surfaces des lenteurs. La seule différence est que ces surfaces sont dispersives, c'est-à-dire qu'elles sont différentes selon la fréquence. On a vu au §1 que les valeurs propres de la matrice de transfert $[\Phi]$ d'une supercouche sont reliées aux lenteurs de Floquet associées aux nombres d'onde de Floquet par une relation exponentielle. On peut donc tracer l'argument $\theta$ d'une valeur propre, c'est-à-dire la projection sur l'axe d'empilement des couches du vecteur nombre d'onde de Floquet multiplié par l'épaisseur totale d'une supercouche, pour une incidence donnée en fonction de la fréquence. Pour une incidence de $10^{\circ}$ dans l'eau, la figure 1 présente les courbes de dispersion d'un multicouche périodique infini de type Carbone-Epoxyde $0^{\circ} / 90^{\circ}$ dont chaque couche est à $90^{\circ}$ de la précédente. Le plan d'incidence étant un plan de symétrie, il y a propagation de quatre ondes de Floquet. Jusqu'à $2.5 \mathrm{MHz}, \theta=\mathrm{f}(\omega)$ sont des droites. Le milieu peut donc être considéré comme homogène. Par ailleurs, à $10^{\circ}$, angle supérieur au premier angle critique dans la première couche du composite, on obtient quatre courbes. Les quatre ondes de Floquet dans le multicouche sont donc propagatives : il y a recomposition des ondes planes classiques dont certaines sont inhomogènes dans la première couche pour donner des ondes de Floquet qui sont toutes propagatives.

Par analogie avec la propagation d'un ébranlement le long d'une chaîne d'atomes séparés par des ressorts représentant les forces de liaison entre atomes $[8,9,10]$, on peut considérer les courbes jusqu'à $5 \mathrm{MHz}$, c'est-à-dire jusqu'à la première bande d'arrêt en fréquence, comme des branches acoustiques, chaque branche correspondant à un mode longitudinal ou à un mode transversal. Les vitesses de propagation des ondes de Floquet peuvent être déduites des pentes des courbes. Toujours par analogie, on peut considérer les courbes à partir de $6 \mathrm{MHz}$ comme des branches optiques.

Pour une fréquence donnée, on peut tracer les variations des lenteurs de Floquet en fonction de l'angle d'incidence dans le milieu multicouche. Un développement grandes longueurs d'onde effectué par T. Lhermitte [11] pour des matériaux $0^{\circ} / 90^{\circ}$ donne des constantes équivalentes. Pour un multicouche de type Carbone-Epoxyde $0^{\circ} 190^{\circ}$, la figure 2 présente les surfaces des lenteurs de Floquet pour une fréquence incidente allant jusqu'à $2.5 \mathrm{MHz}$. Ces surfaces sont exactement celles obtenues en traçant de manière classique les surfaces des lenteurs du matériau homogénéisé dont les constantes élastiques ont été calculées par un développement grandes longueurs d'onde. On a vu au $\$ 2$ que le vecteur polarisation de Floquet varie selon la position dans le multicouche. Dans le domaine des grandes longueurs d'onde, la polarisation de l'onde de Floquet $(\beta)$ tend vers une limite qui n'est autre que la polarisation de l'onde plane classique se propageant dans le milieu homogénéisé. Ainsi, bien que les surfaces des lenteurs du matériau $0^{\circ} / 90^{\circ}$ soient identiques en basse fréquence à celles du matériau homogénéisé, les polarisations de Floquet aux différentes interfaces ne sont pas tout à fait égales (à une constante complexe de module 1 près) aux polarisations du milieu homogénéisé. Le critère d'identité des surfaces des lenteurs [12] n'est donc pas tout à fait suffisant sur le plan de l'homogénéisation. 


\section{4- CONCLUSIONS}

Afin de mieux comprendre la correspondance entre les ondes planes classiques se propageant dans chaque couche du multicouche et les ondes de Floquet, combinaisons linéaires de ces dernières, nous avons défini un vecteur polarisation de Floquet, combinaison linéaire des polarisations dans chaque couche du multicouche, différent selon la position dans ce multicouche et complexe, même si l'onde de Floquet correspondante est propagative. La notion de milieu équivalent au milieu multicouche prend alors un sens plus fort et on peut tracer des courbes de dispersion et des surfaces des lenteurs, fonctions de la fréquence. En basse fréquence, le vecteur polarisation de Floquet tend vers le vecteur polarisation de l'onde se propageant dans un milieu homogénéisé.

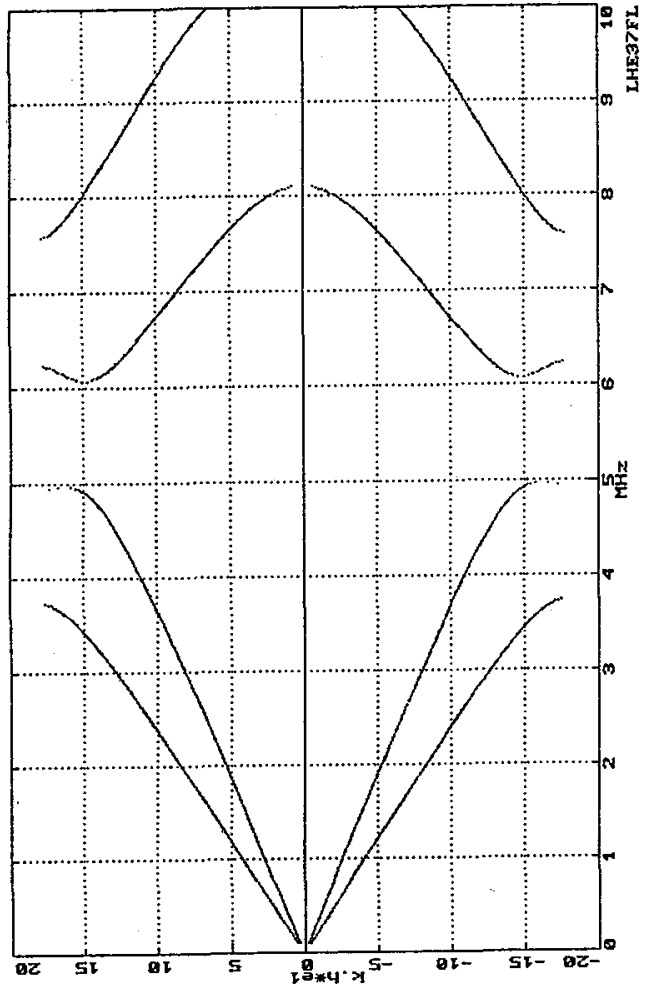

figure 1 : courbes de dispersion pour un $0^{\circ} \% 0^{\circ}$ à $10^{\circ}$

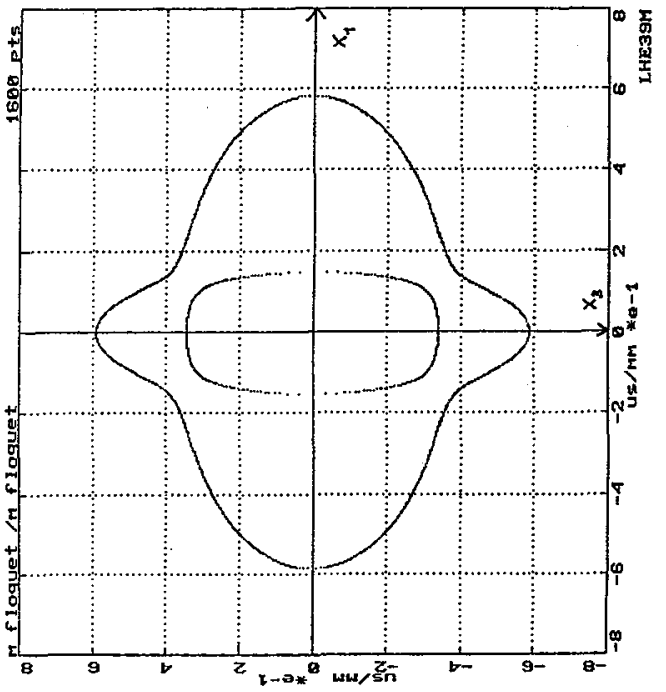

figure 2 : surfaces des lenteurs de Floquet

\section{RÉFÉRENCES BIBLIOGRAPHIQUES}

F. Gilbert, G.E. Backus, Geophysics, 31, 2, (1966), 326-332

A.H. Nayfeh, J. Ac. Soc. Am, 89,4, 1521-1531, (1991)

D.E. Chimenti, A.H. Nayfeh, J. of NDE,9,213, 51-69, (1990)

T.C.T. Ting, P. Chadwick, Wave Prop. in Struc. Comp., 69-79, (Am. Soc. Mec. Eng., New-York, 1988), AMD 90, A.K. Mal and T.C.T. Ting (Eds)

A.M.B. Braga, G. Hermann, J. Ac. Soc. Am.,91,3, (1992), 1211-1227

C. Potel, J.F. de Belleval, J. Ac. Soc. Am,93,5,(1993), 2669-2677

C. Potel, J.F. de Belleval, J. Ap. Phys., 74,4,(1993), 2208-2215

L. Brillouin, Wave propagation in periodic structures, Dover Publ., Inc., 2nd Ed. (1953)

E. Dieulesaint, D. Royer; Ondes élastiques dans les solides; Masson et Cie Eds (1974)

B. de Halleux, A. Kheder, Rev. of Prog. in Quant. NDE, 10, 1951-1958, (1991)

T. Lhermitte, Thèse de doctorat, Université P. et M. Curie de Paris, (1991)

M. Schoenberg, Wave Motion, 6, 302-320, (1984) 